\title{
Comparison of the diagnostic ability of blue laser imaging magnification versus pit pattern analysis for colorectal polyps
}

\section{(ㅇ) $\circledast \Theta$}

\author{
Authors \\ Arihiro Nakanoํㅜㄹ Yoshiki Hirooka², Takeshi Yamamura², Osamu \\ Watanabe ${ }^{1}$, Masanao Nakamura ${ }^{1}$, Kohei Funasaka' ${ }^{1}$, Eizaburo Ohno', \\ Hiroki Kawashima ${ }^{1}$, Ryoji Miyahara ${ }^{1}$, Hidemi Goto ${ }^{1}$ \\ Institutions \\ 1 Department of Gastroenterology and Hepatology, Nagoya \\ University Graduate School of Medicine, Showa-ku, Nagoya, \\ Japan \\ 2 Department of Endoscopy, Nagoya University Hospital, \\ Showa-ku, Nagoya, Japan \\ submitted 14.9.2016 \\ accepted after revision $\quad 30.12 .2016$ \\ Bibliography \\ DOI http://dx.doi.org/10.1055/s-0043-102400 | \\ Endoscopy International Open 2017; 05: E224-E231 \\ (c) Georg Thieme Verlag KG Stuttgart · New York \\ ISSN 0013-726X \\ Corresponding author \\ Takeshi Yamamura, Department of Endoscopy, Nagoya University \\ Hospital, 65 Tsurumai-cho, Showa-ku, Nagoya 466-8550, Japan \\ tyamamu@med.nagoya-u.ac.jp
}

\begin{abstract}
Background and study aims There have been few evaluations of the diagnostic ability of new narrow band light observation blue laser imaging (BLI). The present prospective study compared the diagnostic ability of BLI magnification and pit pattern analysis for colorectal polyps.

Patients and methods We collected lesions prospectively, and the analysis of images was made by two endoscopists, retrospectively. A total of 799 colorectal polyps were examined by BLI magnification and pit pattern analysis at Nagoya University Hospital. The Hiroshima narrow-band imaging classification was used for BLI. Differentiation of neoplastic from non-neoplastic lesions and diagnosis of deeply invasive submucosal cancer (dSM) were compared between BLI magnification and pit pattern analysis. Type $\mathrm{C} 2$ in the Hiroshima classification was evaluated separately, because application of this category as an index of the depth of cancer invasion was considered difficult.

Results We analyzed 748 colorectal polyps, excluding 51 polyps that were inflammatory polyps, sessile serrated adenoma/polyps, serrated adenomas, advanced colorectal cancers, or other lesions. The accuracy of differential diagnosis between neoplastic and nonneoplastic lesions was $98.4 \%$ using BLI magnification and $98.7 \%$ with pit pattern analysis. In addition, the diagnostic accuracy of BLI magnification and pit pattern analysis for dSM for cancer was $89.5 \%$ and $92.1 \%$, respectively. When type C2 lesions were excluded, the diagnostic accuracy of BLI for dSM was $95.9 \%$. The 18 type C2 lesions comprised 1 adenoma, 9 intramucosal or slightly invasive submucosal cancers, and $8 \mathrm{dSM}$. Pit pattern analysis allowed accurate diagnosis of the depth of invasion in 13 lesions (72.2\%).

Conclusions Most colorectal polyps could be diagnosed accurately by BLI magnification without pit pattern analysis, but we should add pit pattern analysis for type C2 lesions in the Hiroshima classification.
\end{abstract}

\section{Introduction}

Endoscopic diagnosis and treatment of colorectal polyps is improving. Performing optical biopsy by image-enhanced endoscopy might reduce the cost and time required for resection and histopathological diagnosis of many diminutive colorectal polyps $[1,2]$. Under such circumstances, more accurate, clinically acceptable, and simple procedures are needed for widespread application of endoscopic approaches such as EMR and endoscopic submucosal dissection (ESD). Pit pattern analysis was reported to be useful for differentiating neoplastic from non-neoplastic colorectal polyps and for evaluating the depth of tumor invasion [3-5]. In addition, narrow-band imaging (NBI; Olympus Co., Tokyo, Japan) can be used to diagnose colorectal polyps and assess the depth of invasion [6-20] with a shorter examination time compared to chromoendoscopy, which has led to its clinical application [21]. However, NBI is inferior to pit pattern analysis in some respects with regard to diagnosis of colorectal polyps $[9,16,19]$. The surface pattern evaluated by $\mathrm{NBI}$ is different from the pit pattern since it is combined with crypt openings and marginal crypt epithelium, so the surface pattern is blurrier than the pit pattern. Fujifilm recently developed a new endoscopic system ("LASEREO") that combines 2 types of laser light with phosphors to allow narrowband light observation blue laser imaging (BLI). The LASEREO consists of a white light laser (peak wavelength: $450 \pm 10 \mathrm{~nm}$ ) that excites phosphors to create white light illumination with broader spectral distribution suitable for normal observation, and a short-wavelength narrow-band laser (wavelength: $410 \pm$ $10 \mathrm{~nm}$ ) to provide information about microvessels and surface 

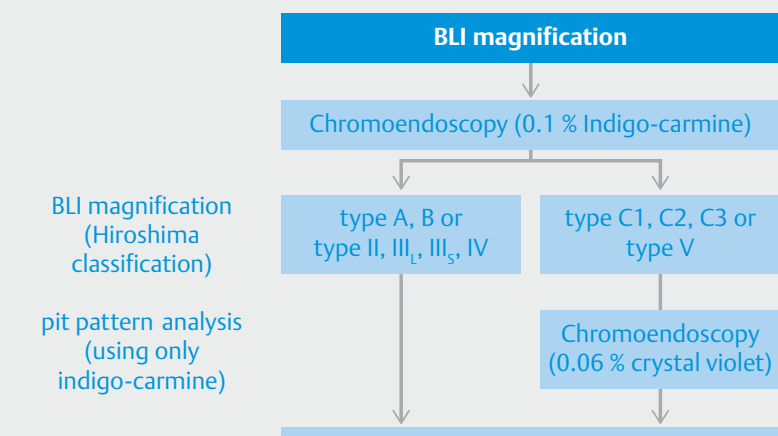

Histopathological examination

Fig. 1 Process of endoscopic examination.

structures as high-contrast signals. Changing the intensity ratios of these two lasers creates illumination that is suitable for either normal or narrow-band observation. In addition, it is easy to obtain brighter images with BLI than NBI using optical filters. Yoshida et al. reported that the diagnostic ability of BLI and NBI for colorectal polyps is equivalent $[22,23]$, but these techniques have not been compared with pit pattern analysis. Accordingly, this study was performed to compare the diagnostic ability of BLI magnification and pit pattern analysis for colorectal polyps, for which both methods are sometimes used concomitantly, and to evaluate a strategy for diagnostic image-enhanced endoscopy (IEE).

\section{Patients and methods}

\section{Patients}

We collected lesions prospectively. A total of 799 colorectal polyps in 506 patients were examined by BLI magnification, pit pattern analysis, and histopathological diagnosis at Nagoya University Hospital between June 2013 and March 2015. In this study, we only evaluated hyperplastic polyps, adenomas, and early colorectal cancers, because lesions such as inflammatory polyps, sessile serrated adenoma/polyps, and serrated adenomas are considered difficult to differentiate even by chromoendoscopy using basal pit pattern classification [24]. The ethics committee of Nagoya University Hospital approved the protocol of this prospective study and all patients provided written informed consent. This study was registered with the University Hospital Medical Information Network Clinical Trials Registry (UMIN-CTR: UMIN 000012348).

\section{Endoscopic examination}

Patients were administered 1 to $2 \mathrm{~L}$ of polyethylene glycol (Niflec $^{\circledR}$ or Moviprep ${ }^{\circledR}$; Ajinomoto Pharma Co., Tokyo, Japan) on the morning of or the night before examination. All patients were examined with an EC-L590ZW magnifying endoscope that had the new LASEREO illumination endoscopic system allowing BLI observation. Examinations were performed by 12 colonoscopists who had total experience with over 3000 colonoscopies and 100 studies using IEE magnification. Polyps de- tected by conventional colonoscopy were observed using BLI magnification, followed by chromoendoscopy using $0.1 \%$ indigo-carmine dye with or without $0.06 \%$ crystal violet dye ( Fig. 1). Crystal violet dye was used only for the lesions with type $\mathrm{C}$ in Hiroshima classification or type $\mathrm{V}$ in pit pattern diagnosis. The number of images was not specified because it differed according to polyp size and morphology.

Biopsy, endoscopic resection (polypectomy, EMR, ESD), or surgical resection was done for all polyps and all cancers were totally resected. Biopsies were obtained from sites of suspected cancer according to findings of the 2 imaging modalities, but were not otherwise specified (only 26 polyps $\geq 10 \mathrm{~mm}$ were biopsied). The biopsy samples were fixed in $10 \%$ formalin, and histopathological diagnosis was done according to World Health Organization (WHO) criteria. In this study, we defined cancer invading the submucosa to a depth $<1,000 \mu \mathrm{m}$ or $\geq 1,000 \mu \mathrm{m}$ as slightly invasive submucosal cancer (sSM) or deeply invasive submucosal cancer (dSM), respectively. Curative endoscopic resection and surgical resection are respectively indicated for SSM and dSM according to the probability of lymph node metastasis $[25,26]$. The depth of submucosal invasion was measured according to the 2014 guidelines of the Japanese Society for Cancer of the Colon and Rectum [26].

\section{Classification}

Several NBI classifications of colorectal polyps have been proposed, which can be separated into those evaluating vascular patterns and those evaluating both surface and vascular patterns $[6,12,18]$. It was thought that BLI could provide images with higher contrast because of improved brightness and narrow spectral bandwidth, so we took advantage of this to employ the Hiroshima classification, which is the only one that evaluates both surface and vascular patterns. According to the Hiroshima classification, lesions are classed as type A, B, or C ( Fig.2). Type $A$ is non-neoplastic lesions, which are hyperplastic polyps (HP) and inflammatory polyps. Type B is adenoma (Ad). Type $C$ is cancer, and this is type divided into three subtypes for differentiating dSM. Type C1 is intramucosal cancer (M) or sSM and type C3 is dSM, while type C2 is reportedly difficult to use for assessing tumor depth $[6,11]$. However, we decided that type C2 indicates M-sSM to compare the diagnostic ability of BLI magnification and pit pattern analysis.

We used the classification of Kudo and Tsuruta for pit pattern analysis [3-5]. Types I and II are non-neoplastic lesions, type III (III, $\left.\mathrm{III}_{\mathrm{S}}\right)$ and type IV are adenomas, and type $\mathrm{V}$ is cancer. Type $V$ is divided into 3 subtypes: $V_{\text {l }}$ low irregularity $\left(V_{1}\right.$ low), $V_{\text {I }}$ high irregularity $\left(\mathrm{V}_{1}\right.$ high), and $\mathrm{V}_{\mathrm{N}}$. $\mathrm{V}_{1}$ low corresponds to M-sSM lesions, while $\mathrm{V}_{\mathrm{l}}$ high and $\mathrm{V}_{\mathrm{N}}$ correspond to $\mathrm{dSM}$ lesions.

\section{Image evaluation}

All images were stored as digital data, separated into BLI and pit pattern images, and randomly evaluated twice each by two expert endoscopists (A.N. and T.Y.) who were blinded to patient background data and their colonoscopic and histopathological findings, retrospectively. They were 2 of the 12 endoscopists who performed colonoscopy in this study and they had experience with over 300 observations using IEE magnification. These 

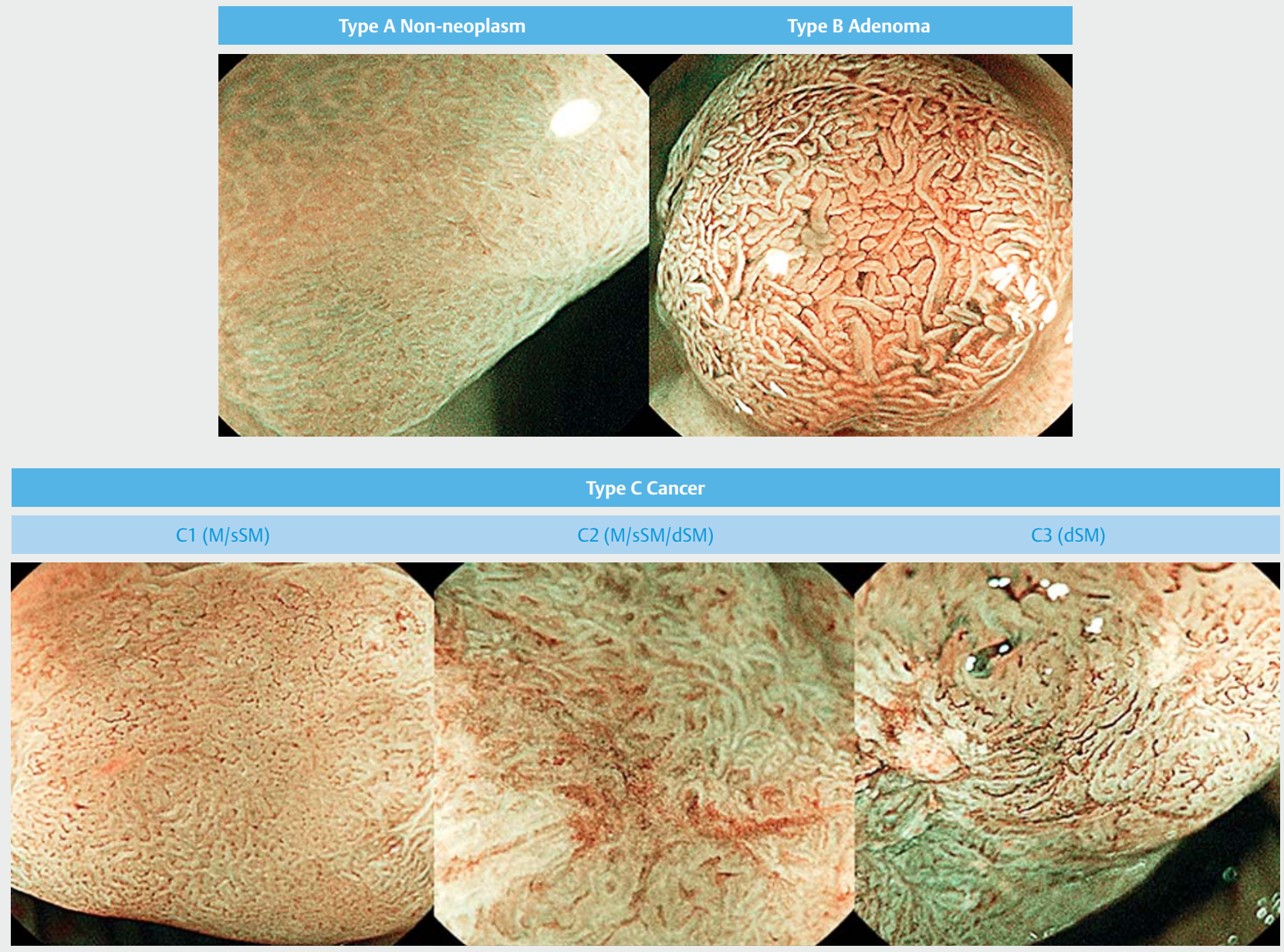

- Fig. 2 Hiroshima classification of blue laser imaging (BLI) magnification for colorectal polyps. Type A is non-neoplastic lesions: microvessels are vague or invisible. Type B is adenoma: regular surface pattern with increased microvessel intensity around pits and regular microvessel mesh. Type $C$ is cancer, and is divided into three subtypes. Type $\mathrm{C} 1$ is intramucosal cancer (M) or slightly invasive submucosal cancer (sSM): irregular surface pattern with vessels of homogeneous thickness and distribution. Type C2 is M or sSM or deeply invasive submucosal cancer (dSM): obviously irregular surface pattern and irregular vessels of heterogeneous thickness and distribution. Type C 3 is dSM: invisible surface pattern with avascular areas.

expert endoscopists evaluated images under the same conditions and excluded unappraisable images to assess the usefulness of BLI magnification precisely. The evaluation criteria for images generated by each modality were standardized between the two endoscopists before assessment. Conventional colonoscopic images were not used, so that their effect on the diagnosis made using each modality was eliminated. The second evaluation was performed one month or more after the first evaluation, with images of each lesion being evaluated twice each by the two endoscopists. The order of evaluation was all BLI images followed by all pit pattern images. The images of each lesion were evaluated twice each by the 2 endoscopists. If the interpretation was in agreement on 3 or 4 evaluations, it was adopted. If the interpretation was in agreement on 1 or 2 evaluations, a conclusion was reached by a conference between the 2 endoscopists. The intraobserver and interobser- ver agreement rates were analyzed separately for BLI and pit pattern analysis.

\section{Study outcomes}

We analyzed the relationship between the histopathological diagnosis and BLI magnification interpreted by the Hiroshima classification or pit pattern analysis. The diagnostic accuracy, rate of differentiating neoplastic from non-neoplastic lesions, and diagnosis of deeply invasive submucosal cancer (dSM) were compared between BLI magnification and pit pattern analysis. Type C2 in the Hiroshima classification was evaluated separately because application of this category to assess the depth of tumor invasion is considered to be difficult. 


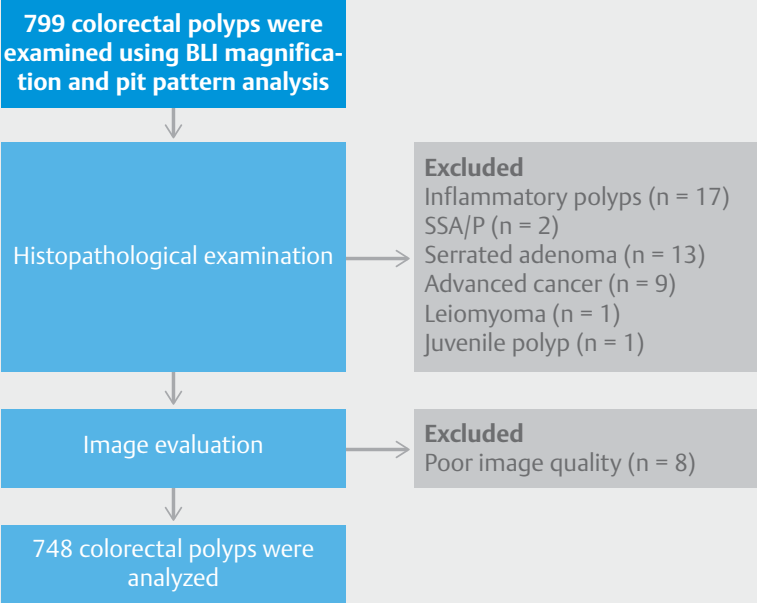

Fig. 3 Lesions excluded from this study.

\section{Statistical analysis}

The diagnostic ability of BLI magnification and pit pattern analysis were compared by using the $x^{2}$-test and Fisher's exact test, with $P<0.05$ being considered statistically significant. The rates of intraobserver and interobserver agreement with respect to image interpretation were calculated as $\mathrm{k}$ values. We defined a $\mathrm{K}$ value of $<0.4,0.41-0.60,0.61-0.80$ and $\geq 0.81$ as poor, fair, good, and excellent agreement, respectively. All data were analyzed using SPSS software (version 22).

\section{Results}

Among 799 colorectal polyps examined by BLI magnification, pit pattern analysis, and histopathological analysis, the inflammatory polyps $(n=17)$, sessile serrated adenomas/polyps $(n=$ $2)$, and serrated adenomas $(n=13)$ were excluded, along with exclusion of advanced colorectal cancers $(n=9)$, submucosal tumor (leiomyoma: $n=1$ ), and juvenile polyp $(n=1)$. Lesions that the two expert endoscopists considered difficult to diagnose because of poor image quality were also excluded $(n=8)$ ( $\triangleright$ Fig.3). The remaining 748 colorectal polyps were analyzed.

- Table 1 shows the details of 748 lesions, which were classified as HP ( $n=66), \operatorname{Ad}(n=568), M-s S M(n=94)$, and dSM ( $n=$ 20). Histological specimens of 316, 407 and 25 lesions were obtained by biopsy, endoscopic resection, and surgery, respectively. Comparison between BLI magnification, pit pattern analysis, and histopathological diagnosis is shown in $>$ Table 2 . The overall diagnostic accuracy of BLI magnification was $89.3 \%$ and that of pit pattern analysis was $91.4 \%$.

According to BLI magnification and histopathological diagnosis, $93.5 \%$ of type A lesions were non-neoplastic, while $98.8 \%$ of type B, C1, C2, and C3 lesions were neoplasms. The accuracy, sensitivity, and specificity of differential diagnosis between neoplastic and non-neoplastic lesions was $98.4 \%$, $87.9 \%$, and $99.4 \%$, respectively. According to pit pattern analysis, $91.2 \%$ of type II lesions were non-neoplastic, while $99.4 \%$ of type $I I I_{L}$, III $, I V, V_{1}$ low, $V_{1}$ high, and $V_{N}$ lesions were neoplasms. The accuracy, sensitivity, and specificity of making a differential diagnosis between neoplastic and non-neoplastic lesions was $98.7 \%, 93.9 \%$, and $99.1 \%$, respectively.

Regarding the differential diagnosis of dSM for cancers, the accuracy, sensitivity, and specificity was $89.5 \%, 40.0 \%$, and $100 \%$ by BLI magnification, and $92.1 \%, 75.0 \%$, and $95.7 \%$ by pit pattern analysis, respectively. When type $C 2$ lesions were excluded, the accuracy, sensitivity and specificity of differential diagnosis of dSM was $95.9 \%, 66.7 \%$, and $100 \%$, respectively. - Table 3 compares the diagnoses made by the two modalities. There were high rates of agreement between types A, B, and C3 and the corresponding pit patterns, whereas agreement with type $\mathrm{C} 1$ was slightly lower and varied like the agreement rate with histopathological diagnosis. The rate of agreement with the histopathological diagnosis between BLI magnification and pit pattern analysis was $92.0 \%$.

\begin{tabular}{|c|c|}
\hline \multicolumn{2}{|l|}{ Patients $(n=481)$} \\
\hline " Male/female, no. & $306 / 175$ \\
\hline - Age, years, mean (range) ( \pm SD) & $66.9(30-91)(10.7)$ \\
\hline \multicolumn{2}{|l|}{ Polyps $(n=748)$} \\
\hline - Location (right ${ }^{1} /$ left $^{2} /$ rectum) & $395 / 252 / 101$ \\
\hline " Mean size (range) $( \pm S D)(m m)$ & $10.7(2-175)(12.4)$ \\
\hline - Morphology (protruded/superficial) & $541 / 207$ \\
\hline $\begin{array}{l}\text { - Procedure of getting specimens } \\
\text { (biopsy/endoscopic resection/surgical resection) }\end{array}$ & $316 / 407 / 25$ \\
\hline $\begin{array}{l}\text { - Histopathology } \\
\text { (HP/Ad/M-sSM/dSM) }\end{array}$ & $66 / 568 / 94 / 20$ \\
\hline \multicolumn{2}{|c|}{$\begin{array}{l}\text { SD, standard deviation; HP, hyperplastic polyp; Ad, adenoma; M, intramucosal cancer; sSM, slightly invasive submucosal cancer; dSM, deeply invaded submucosa } \\
\text { cancer } \\
{ }^{1} \text { Right : cecum, ascending colon, and transverse colon } \\
{ }^{2} \text { Left : descending colon and sigmoid colon }\end{array}$} \\
\hline
\end{tabular}


- Table 2 a Comparison between BLI magnification and histopathological diagnosis.

\begin{tabular}{|c|c|c|c|c|c|}
\hline \multirow{2}{*}{$\begin{array}{l}\text { BLI } \\
\text { Hiroshima classification }\end{array}$} & \multirow[b]{2}{*}{ No. lesions } & \multicolumn{4}{|c|}{ Histopathological diagnosis } \\
\hline & & HP & Ad & M-sSM & dSM \\
\hline A & 62 & 58 & 4 & & \\
\hline B & 563 & 8 & 531 & 23 & 1 \\
\hline C1 & 97 & & 32 & 62 & 3 \\
\hline C2 & 18 & & 1 & 9 & 8 \\
\hline C3 & 8 & & & & 8 \\
\hline Total & 748 & 66 & 568 & 94 & 20 \\
\hline
\end{tabular}

- Table $\mathbf{2}$ b Comparison between pit pattern analysis and histopathological diagnosis.

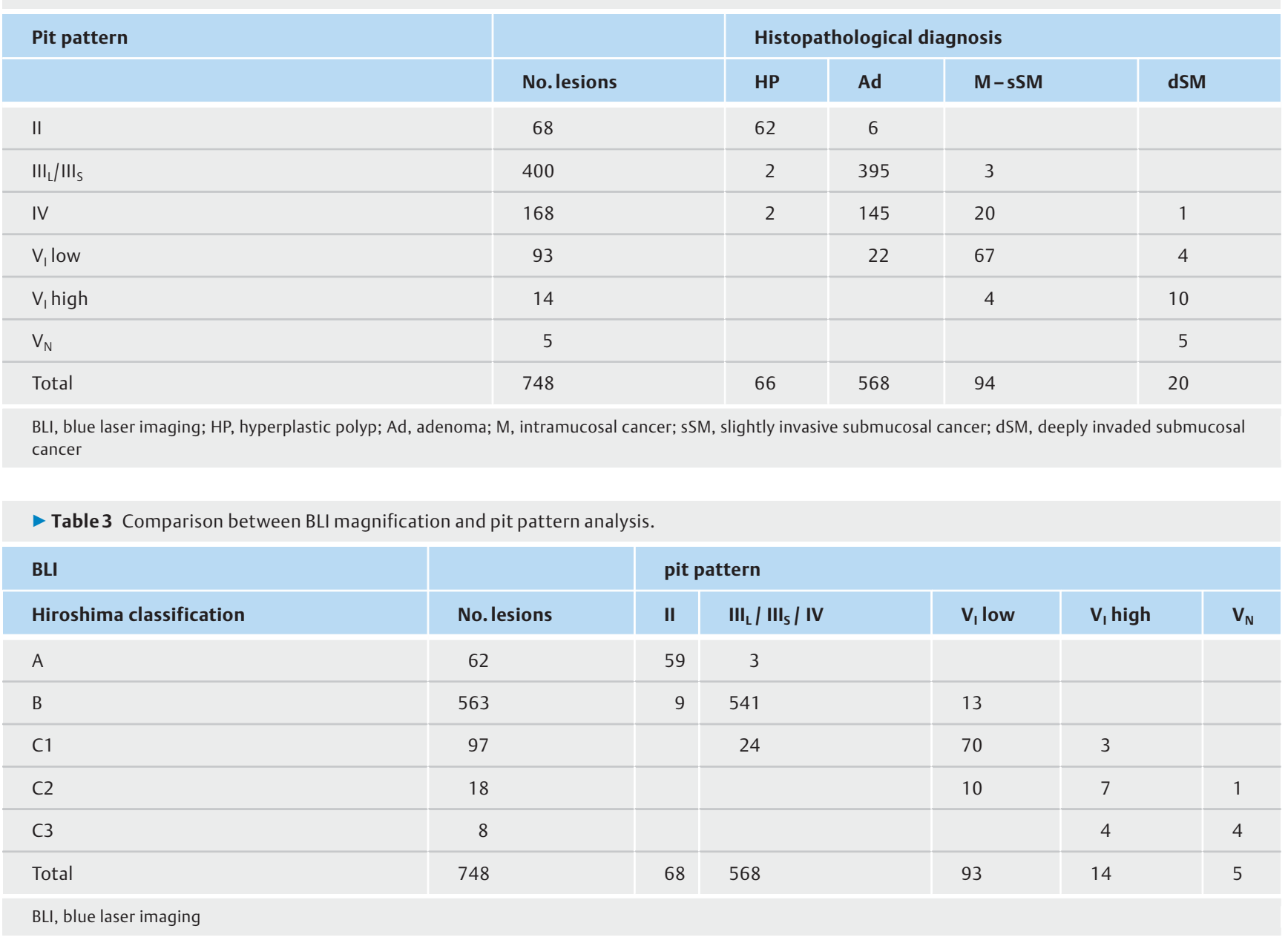

Among 18 lesions classified as type C2 by BLI magnification ( $>$ Fig.4), 10 were $V_{1}$ low, 7 were $V_{1}$ high, and 1 was $V_{N}$ by pit pattern analysis. The depth of invasion was accurately diagnosed for 13 (72.2\%) of the 18 lesions.
Interobserver agreement about the diagnosis of colorectal polyps by each modality was good (BLI, $\mathrm{K}=0.617-0.659$; pit pattern, $\mathrm{k}=0.621-0.743)$, and intraobserver agreement was also good for both BLI magnification (A. N., $\mathrm{K}=0.767$; T.Y., $\mathrm{k}=$ 0.760 ) and pit pattern analysis (A. N., $\mathrm{K}=0.745$; T. Y., $\mathrm{K}=0.773$ ). 
BLI magnification (Hiroshima classification)

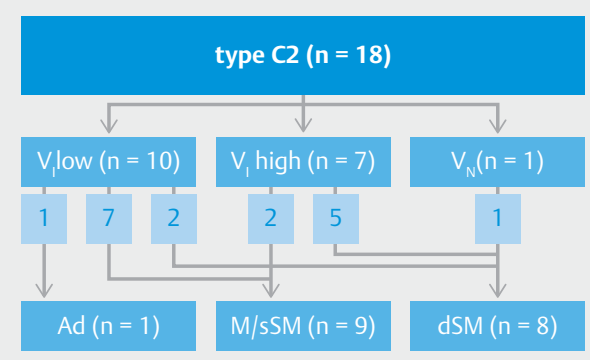

Histopathological diagnosis

- Fig. 4 Comparison of pit pattern analysis and histopathological diagnosis of type C2 lesions.

\section{Discussion}

Endoscopic treatment is indicated for colorectal lesions that might become cancerous (suspected cancers) and for cancers without metastasis (or a low risk of metastasis) $[25,26]$. The method of endoscopic resection is determined according to tumor morphology and size $[27,28]$ making accurate preoperative diagnosis clinically important. In addition, the method selected should preferably be tolerated well by the patient (simple and rapid) and less invasive, if possible. Pit pattern analysis is an established and highly reliable diagnostic technique [35]. However, spraying dyes such as indigo carmine and crystal violet requires troublesome preparation and implementation, and also has disadvantages such as interference with lesion resection and post-spraying visualization of surrounding structures. In contrast, normal light observation can be switched instantaneously to NBI and BLI without such disadvantages [21].

Recently, the new NBI magnifying endoscopic classification of colorectal tumors proposed by the Japan NBI Expert Team (JNET) [29]. Both the JNET classification and Hiroshima classification evaluates vascular patterns and surface pattern. Type C1 and $C 3$ in Hiroshima classification are correlated with the type 2B and type 3 in JNET classification. The most of type C2 in Hiroshima classification is correlated type $2 \mathrm{~B}$, and the part of that is correlated type 3 in JNET classification. By dividing lesions of type C2, we may narrow down lesions which need chromoendoscopy. The criterion of type C2 in Hiroshima classification lacks in objectivity. We suggest that further investigation of type $\mathrm{C} 2$ in Hiroshima classification may diagnose colorectal polyps more accurately. Additionally, Yoshida et al. reported that the ability of BLI diagnosis were similar to that of NBI using Hiroshima classification for colorectal polyps. On the other hands, the ability of BLI using JNET classification has not been evaluated.

Using BLI magnification, the accuracy of differentiating between neoplastic and non-neoplastic lesions was $98.4 \%$, the diagnostic accuracy was $89.3 \%$ using histopathological diagnosis as the standard, and the accuracy of differential diagnosis of dSM for cancers was $89.5 \%$. With simultaneous pit pattern analysis, the accuracy of differentiating between neoplastic and non-neoplastic lesions was $98.7 \%$, the diagnostic accuracy compared with histopathological diagnosis was $91.4 \%$, and the accuracy of differential diagnosis of dSM for cancers was $92.1 \%$. Thus, the results obtained with BLI magnification and pit pattern analysis were highly similar. Additionally, the accuracy of differential diagnosis for dSM was improved by separating type C2 lesions. Yoshida et al. found that the diagnostic accuracy of BLI magnification for colorectal polyps was $84.3 \%$, the accuracy of differentiating between neoplastic and non-neoplastic lesions was $99.3 \%$, and the accuracy of differential diagnosis of dSM was $94.3 \%$ [22]. The same authors stated that the diagnostic ability of BLI and NBI magnification is equivalent to histopathological diagnosis [23]. Others have reported that the accuracy of differentiating between neoplastic and nonneoplastic lesions by NBI magnification is $95.3 \%$ to $98.2 \%$, which are results close to that obtained in this study (98.4\%) $[2,12,14,16,17]$.

dSM lesions accounted for $0 \%$ of type A, $0.17 \%$ of type B, $3.1 \%$ of type $C 1,44.4 \%$ of type $C 2$, and $100 \%$ of type $C 3$ according to BLI magnification, while these lesions accounted for $0 \%$ of type II, $0.17 \%$ of types $I I_{L}$, III , and IV, $4.3 \%$ of type $V_{\text {I low, }}$ $71.4 \%$ of type $V_{1}$ high, and $100 \%$ of type $V_{N}$ according to pit pattern analysis. These data also show that about half of the lesions judged to be type $\mathrm{C} 2$ by BLI magnification were dSM, indicating that the $\mathrm{C} 2$ category cannot be used as an index of the depth of tumor invasion. In contrast, pit pattern analysis accurately diagnosed the depth of invasion for $72.2 \%(13 / 18)$ of type C2 lesions in the present study. Therefore, the accuracy, sensitivity, and specificity of the differential diagnosis of dSM lesions was respectively $98.9 \%, 70.0 \%$, and $99.7 \%$ when pit pattern analysis was applied to type C2 lesions of the Hiroshima classification.

Many polyps are considered to be targets for treatment to prevent colorectal cancer, but this requires considerable effort and cost. On the other hand, Løberg et al. reported that colorectal cancer mortality was lower among patients who had low-risk adenomas removed compared with the general population regardless of polyp size and number [30]. Therefore, narrowing the gap between endoscopic diagnosis and histopathological diagnosis, which is the gold standard, is critical for reducing the physical burden on medical professionals and patients as well as the cost of health care. The diagnostic ability of BLI magnification and pit pattern analysis was comparable in the present study, suggesting that these examinations can contribute to judgment about the need for resection and surveillance of polyps.

While BLI is clearly useful, the results did not significantly surpass those of other observation techniques using lightbased technologies. However, BLI can theoretically provide images with better contrast than other narrow band techniques, so further image analysis and comparison with other diagnostic methods are needed to widen its applicability.

This study had several limitations. First, we diagnosed colorectal polyps from still images obtained by BLI magnification without using conventional white light colonoscopic images. Therefore, interpretation was not the same as with real-time endoscopic diagnosis. Second, the number of images in one lesion was not specified. Therefore the number of images might affected the diagnosis. Third, some precancerous lesions (ses- 
sile serrated adenomas/polyps and serrated adenomas) were excluded in our study, although differentiation between these lesions and hyperplastic polyps is important. Further investigation of these lesions will be required. Fourth, the analysis of images were made by only two experts. So, the generalizability of the result was not so high. Additionally, these two endoscopists evaluated some images they collected before, therefore, they might remember some images of the lesions. Finally, a part of lesions were not resected. Histological specimens of these lesions were obtained by biopsy. All lesions should be resected for correctly diagnosis.

\section{Conclusions}

In conclusion, the diagnostic ability of BLI magnification and pit pattern analysis for colorectal polyps was comparable and both methods showed great clinical applicability. To maximize the advantages of BLI magnification and complement its disadvantages, pit pattern analysis (dye spraying) should be omitted for type A, B, C1, and C3 lesions in the Hiroshima classification and should be selectively applied to type $C 2$ lesions. This provides a simple, acceptable, and highly accurate strategy for diagnosis of colorectal polyps.

\section{Acknowledgements}

The authors would like to thank Yamada K, Nagura A, Yoshimura T, Oshima H, Saito M, Matsuura R, Mizutani Y, and Sawada $\mathrm{T}$ for performing colonoscopy and collecting images.

\section{Competing interests}

Takeda Pharmaceutical Co., Ltd., Eisai Co., Ltd., Daiichi Sankyo Co., Ltd, Otsuka Pharmaceutical Co., Ltd., CHUGAI PHARMACEUTICAL CO.,LTD., Merck \& Co., Inc.(Merck Sharp \& Dohme (MSD), Sumitomo Dainippon Pharma Co., Ltd., TAlHO Pharmaceutical Co., Ltd., Mitsubishi Tanabe Pharma Corporation, KYORIN Pharmaceutical Co., Ltd., Kyowa Hakko Kirin Co., Ltd., AbbVie GK.

\section{References}

[1] Kaltenbach T, Rastogi A, Rouse RV et al. Real-time optical diagnosis for diminutive colorectal polyps using narrow-band imaging: the VALID randomised clinical trial. GUT 2015; 64: 1569-1577

[2] Ingiatovic A, East JE, Suzuki $\mathrm{N}$ et al. Optical diagnosis of small colorectal polyps at routine colonoscopy (Detect In Spect ChAracterise Resect and Discard; DISCARD trial): a prospective cohort study. Lancet Oncol 2009; 10: 1171-1178

[3] Kudo S, Hirota S, Nakajima T et al. Colorectal tumors and pit pattern. J Clin Pathol 1994; 47: 880-885

[4] Kudo S, Tamura S, Nakajima T et al. Diagnosis of colorectal tumorous lesions by magnifying endoscopy. Gastrointest Endosc 1996; 44: $8-14$

[5] Kanao H, Tanaka S, Oka S et al. Clinical significance of type V(I) pit pattern subclassification in determining the depth of invasion of colorectal neoplasms. World J Gastroenterol 2008; 14: 211-217
[6] Kanao H, Tanaka S, Oka S et al. Narrow-band imaging magnification predicts the histology and invasion depth of colorectal tumors. Gastrointest Endosc 2009; 69: 631-636

[7] Takata S, Tanaka S, Hayashi $\mathrm{N}$ et al. Characteristic magnifying narrowband imaging features of colorectal tumors in each growth type. Int J Colorectal Dis 2013; 28: 459-468

[8] Hayashi N, Tanaka S, Hewett DG et al. Endoscopic prediction of deep submucosal invasive carcinoma: validation of the Narrow-Band Imaging International Colorectal Endoscopic (NICE) classification. Gastrointest Endosc 2013; 78: 625-632

[9] Hayashi N, Tanaka S, Kanao H et al. Relationship between narrowband imaging magnifying observation and pit pattern diagnosis in colorectal tumors. Digestion 2013; 87: $53-58$

[10] Oba S, Tanaka S, Oka S et al. Characterization of colorectal tumors using narrow-band imaging magnification: combined diagnosis with both pit pattern and microvessel features. Scand J Gastroenterol 2010; 45: $1084-1092$

[11] Oba S, Tanaka S, Sano Y et al. current status of narrow-band imaging magnifying colonoscopy for colorectal neoplasia in Japan. Digestion 2011; 83: 167-172

[12] Uraoka T, Saito Y, Ikematsu H et al. Sano's capillary pattern classification for narrow-band imaging of early colorectal lesions. Dig Endosc 2011; 23: $112-115$

[13] Hirata M, Tanaka S, Oka S et al. Evaluation of microvessels in colorectal tumors by narrow band imaging magnification. Gastrointest Endosc 2007; 66: $945-952$

[14] Wada Y, Kudo SE, Misawa M et al. Vascular pattern classification of colorectal lesions with narrow band imaging magnifying endoscopy. Dig Endosc 2011; 23: 106 - 111

[15] Ikematsu H, Matsuda T, Emura F et al. Efficacy of capillary pattern type IIIA/IIIB by magnifying narrow band imaging for estimating depth of invasion of early colorectal neoplasms. BMC Gastroenterol 2010; 10: 33

[16] Wada Y, Kashida H, Kudo S et al. Diagnostic accuracy of pit pattern and vascular pattern analyses in colorectal lesions. Dig Endosc 2010; 22: $192-199$

[17] Wada $\mathrm{Y}$, Kudo S, Kashida $\mathrm{H}$ et al. Diagnosis of colorectal lesions with the magnifying narrow-band imaging system. Gastrointest Endosc 2009; 70: $522-531$

[18] Saito S, Tajiri H, Ohya T et al. The benefit of using narrow-band imaging systems for observation of capillary networks before determining of treatments for early colon cancer. Dig Endosc 2011; 23: 120 125

[19] Sakamoto T, Saito Y, Nakajima T et al. Comparison of magnifying chromoendoscopy and narrow-band imaging in estimation of early colorectal cancer invasion depth: a pilot study. Dig Endosc 2011; 23 : $118-1123$

[20] McGill SK, Evangelou E, loannidis JP et al. Narrow band imaging to differentiate neoplastic and non-neoplastic colorectal polyps in real time: a meta-analysis of diagnostic operating characteristics. GUT 2013; 62: $1704-1713$

[21] Sakamoto T, Matsuda T, Aoki T et al. Time saving with narrow-band imaging for distinguishing between neoplastic and non-neoplastic small colorectal lesions. J Gastroenterol Hepatol 2012; 27: 351- 355

[22] Yoshida N, Yagi N, Inada Y et al. Ability of a novel blue laser imaging system for the diagnosis of colorectal polyps. Dig Endosc 2014; 26: $250-258$

[23] Yoshida N, Hisabe T, Inada Y et al. The ability of a novel blue laser imaging system for the diagnosis of invasion depth of colorectal neoplasms. J Gastroenterol 2014; 49: $73-80$

[24] Oka S, Tanaka S, Hiyama T et al. Clinicopathologic and endoscopic features of colorectal serrated adenoma: differences between polypoid and superficial types. Gastrointest Endosc 2004; 59: 213-219 
[25] Kitajima K, Fujimori T, Fujii S et al. Correlations between lymph node metastasis and depth of submucosal invasion in submucosal invasive colorectal carcinoma: a Japanese collaborative study. J Gastroenterol 2004; 39: 534-543

[26] Watanabe T, Itabashi M, Shimada Y et al. Japanese Society for Cancer of the Colon and Rectum (JSCCR) Guidelines 2014 for treatment of colorectal cancer. Int J Clin Oncol 2015; 20: 207 - 239

[27] Uraoka T, Saito Y, Matsuda T et al. Endoscopic indications for endoscopic mucosal resection of laterally spreading tumours in the colorectum. Gut 2006; 55: $1592-1597$
[28] Oka S, Tanaka S, Kanao $\mathrm{H}$ et al. Therapeutic strategy for colorectal laterally spreading tumor. Dig Endosc 2009; 21: 43-46

[29] Yasushi S, Tanaka S, Shin-ei K et al. Narrow-band imaging (NBI) magnifying endoscopic classification of colorectal tumors proposed by the Japan NBI Expert Team. Dig Endosc 2016; 28: 526-533

[30] Løberg M, Kalager M, Holme Ø et al. Long-term colorectal cancer mortality after adenoma removal. N Engl J Med 2014; 371: 799-807 